\title{
THE OPTIMAL PORTFOLIO WEIGHTS USING THE PROPORTIONAL TYPE ESTIMATORS
}

\section{Epha Diana Supandi ${ }^{1,2}$, Dedi Rosadi ${ }^{2}$ and Abdurakhman ${ }^{2}$}

${ }^{1}$ Study Program of Mathematics

UIN Sunan Kalijaga

Yogyakarta, Indonesia

e-mail: epha.supandi@uin-suka.ac.id

${ }^{2}$ Department of Mathematics

University of Gadjah Mada

Yogyakarta, Indonesia

e-mail: dedirosadi@ugm.ac.id; rachmanstat@ugm.ac.id

\begin{abstract}
Error estimation in both the expected returns and the covariance matrix hamper the construction of optimal mean-variance portfolio model. In order to overcome this problem, we consider the class of proportional type estimators. The sensitivity of the proposed estimators to errors is measured by the expected loss function (the risk function). The simulation study is conducted when multivariate returns are normally distributed and serially independent. Furthermore, simulation study is complemented by an investigation of the ex post excess returns for empirical datasets, i.e., average, standard deviation, Sharpe ratio, and utility. It turns out that the unbiased proportional estimator and the maximum likelihood estimator are underperformed compared to "the dominant" estimator.
\end{abstract}

Received: September 26, 2016; Accepted: November 9, 2016

2010 Mathematics Subject Classification: 62P20, 91B82.

Keywords and phrases: error estimation, the expected loss function, the proportional type estimators. 


\section{Introduction}

The mean-variance portfolio, which was introduced by Markowitz [12] is one of the standard frameworks employed to determine the optimal portfolio weights. He proposed away to find such a portfolio by analyzing meanvariance structure of the return assets. The mean-variance portfolio assumes that rational investors would choose the lowest risk (measured by variance) at a certain level of expected return or choose the portfolio that gives the highest return at given level of risk.

Mean-variance (MV) portfolio models of Markowitz are formulated mathematically in the following optimization problem:

$$
\begin{aligned}
& \max _{w} \boldsymbol{\mu}^{\prime} \boldsymbol{w}-\frac{\gamma}{2} \boldsymbol{w}^{\prime} \boldsymbol{\Sigma} \boldsymbol{w} \\
& \text { s.t.: } \boldsymbol{e}^{\prime} \boldsymbol{w}=1
\end{aligned}
$$

It is known that $\boldsymbol{\mu} \in \mathfrak{R}^{p}$ is the vector of expected return, $\boldsymbol{\Sigma} \in \mathfrak{R}^{p \times p}$ is the covariance matrix of return, and $\boldsymbol{w} \in \mathfrak{R}^{p}$ is the vector of portfolio weight. The parameter $\gamma$ can be interpreted as a risk aversion, since it takes into account the trade-off between risk and return of the portfolios.

Suppose that $\boldsymbol{w}(\boldsymbol{\mu}, \boldsymbol{\Sigma})$ is the optimal portfolio weights (true optimal portfolio weight) based on $\boldsymbol{\mu}$ and $\boldsymbol{\Sigma}$. In practice, parameters $\boldsymbol{\mu}$ and $\boldsymbol{\Sigma}$ are unknown and must be estimated from historical data sets. We denote random returns of the $p$ risky assets if $\boldsymbol{r}=\left(\boldsymbol{r}_{1}, \boldsymbol{r}_{2}, \ldots, \boldsymbol{r}_{p}\right)^{\prime}$, then the maximum likelihood estimators of $\boldsymbol{\mu}$ and $\boldsymbol{\Sigma}$ are $\hat{\boldsymbol{\mu}}=\overline{\boldsymbol{r}}=1 / n \sum_{1=1}^{n} \boldsymbol{r}_{i}$ and $\hat{\boldsymbol{\Sigma}}=\boldsymbol{S}=$ $1 / n \sum_{i=1}^{n}\left(\boldsymbol{r}_{i}-\overline{\boldsymbol{r}}\right)\left(\boldsymbol{r}_{i}-\overline{\boldsymbol{r}}\right)^{\prime}$, respectively. The maximum likelihood estimator of optimal portfolio is given by $\hat{\boldsymbol{w}}(\overline{\boldsymbol{r}}, \boldsymbol{S})$. However, the optimal portfolio weights obtained by this approach are instable and unreliable.

Estimation of parameters of future asset returns is an essential step in the application of the mean-variance paradigm in practical finance. The impact 
of error estimation on the Markowitz optimization procedure has been intensively analyzed for many years. For example, Best and Grauer [1] analyzed the sensitivity of optimal portfolios to changes in expected return estimates, Chopra and Ziemba [4] showed that even slight changes to the estimates of expected return or risk can produce vastly different meanvariance optimized portfolios. Meanwhile, Broadie [2] showed how the estimated efficient frontier overestimates the expected returns of portfolios for various levels of error estimation, Ceria and Stubbs [3] and Jobson and Korkie [8] have also pointed out this problem. Because of the ill-effects of estimation errors on optimal portfolios, portfolio optimization has been called “error maximization” (see Michaud [13]).

Although many researchers raised serious objections to the meanvariance model as a framework for defining optimal portfolio weight and proposed a number of alternatives, this model serves as the standard optimization framework for modern asset management (see Mori [14]). Thus it is important to examine the problem statistically under the mean-variance model. Few studies have addressed the problem of the mean-variance optimal portfolio weights statistically. Jobson and Korkie [8] and Ledoit [11] derived the asymptotic distribution of $\hat{\boldsymbol{w}}(\overline{\boldsymbol{r}}, \boldsymbol{S})$. Mori [14] and Kinkawa [10] have shown some interesting analytical results under the loss function used in the estimation problem of mean-variance model. Meanwhile, Palczewski and Palczewski [16] derived the closed-form formula related to the stability of portfolio estimator by using a mean square error.

The quadratic loss function is usually used to evaluate the accuracy of an estimator. The loss functions in mean-variance problem are defined as the difference between the utility of the true optimal portfolio and that of an estimated portfolio. For this purpose, we extend the result of Mori [14] by quantification of the impact of the error estimation of the parameters on the portfolio weights both in simulation and empirical studies. Furthermore, we consider the class of proportional type estimators in which the maximum likelihood estimator $\hat{\boldsymbol{w}}(\overline{\boldsymbol{r}}, \boldsymbol{S})$ belongs to this class.

The rest of the paper is organized as follows. In Section 2, we first 
formulate the class of proportional type estimator and present the unbiased estimator of $\boldsymbol{w}(\boldsymbol{\mu}, \boldsymbol{\Sigma})$. In Section 3, we present the definition of the loss function and fundamental results for the proportional type estimator. In Section 4, we show risk behavior of the class proportional type estimator by Monte Carlo simulation and the results of empirical studies. Meanwhile, the conclusion is presented in Section 5.

\section{The Class of Proportional Type Estimators}

The mean-variance portfolio model (equation (1)) can be modeled more generally as a problem solving quadratic programming with linear constraints, namely:

$$
\begin{aligned}
& \max _{w} \boldsymbol{\mu}^{\prime} \boldsymbol{w}-\frac{\gamma}{2} \boldsymbol{w}^{\prime} \mathbf{Q} \boldsymbol{w} \\
& \text { s.t.: } \boldsymbol{A}^{\prime} \boldsymbol{w}=\boldsymbol{b},
\end{aligned}
$$

where $\boldsymbol{Q}$ is a $p \times p$ symmetric and nonsingular matrix, $\boldsymbol{A}$ is a $p \times q$ matrix of rank $q$ and $\boldsymbol{b}$ is a $q \times 1$ vector. Then the optimal solution of the problem (equation (2)) is given by

$$
\boldsymbol{w}(\boldsymbol{\mu}, \boldsymbol{\Sigma})=\frac{1}{\gamma} F_{1}(\boldsymbol{A}, \boldsymbol{Q}) \boldsymbol{\mu}+F_{2}(A, \boldsymbol{Q}) \boldsymbol{b},
$$

where, as in Mori [14], we define:

$$
F_{1}(A, Q)=Q^{-1}-Q^{-1} \boldsymbol{A}\left(\boldsymbol{A}^{\prime} \boldsymbol{Q}^{-1} \boldsymbol{A}\right)^{-1} \boldsymbol{A}^{\prime} \boldsymbol{Q}^{-1}
$$

and

$$
F_{2}(A, Q)=Q^{-1} A\left(A^{\prime} Q^{-1} A\right)^{-1} b .
$$

The class of estimators which is taken into account in this paper is given by Mori [14], i.e., the class of proportional type estimators. According to Mori [14], the definition of the proportional type estimators of $\boldsymbol{w}(\mu, \Sigma)$ is as such. 
Definition 2.1. Let $c$ be a positive constant. Then the following function:

$$
\hat{\boldsymbol{w}}(c, \overline{\boldsymbol{r}}, \boldsymbol{S})=\frac{c}{\gamma} F_{1}(A, \boldsymbol{S}) \overline{\boldsymbol{r}}+F_{2}(A, \boldsymbol{S})
$$

is the proportional type estimator of optimal portfolio weights.

Next, as stated by Mori [14], the unbiased estimator of optimal portfolio weights is given by the following theorem.

Theorem 1 (The unbiased estimator of optimal portfolio weights). Let

$$
\begin{array}{r}
n>\max \{p+1 ; p-q+2\} \text { and } c^{*}=\frac{n-p+q-2}{n} . \text { Then } \\
\hat{\boldsymbol{w}}\left(c^{*}, \overline{\boldsymbol{r}}, \boldsymbol{S}\right)=\frac{c^{*}}{\gamma} F_{1}(\boldsymbol{A}, \boldsymbol{S}) \overline{\boldsymbol{r}}+F_{2}(\boldsymbol{A}, \boldsymbol{S}) \boldsymbol{b}
\end{array}
$$

is an unbiased estimator for $\boldsymbol{w}(\boldsymbol{\mu}, \boldsymbol{\Sigma})$.

\section{The Loss Function of the Optimal Portfolio Weights}

In Section 2, we have made a review of the proportional type estimator for optimal portfolio weights. In this section, we give the definition of the loss function in the estimation problem of the mean-variance optimal portfolio.

We assume that an investor chooses the portfolio weights so as to maximize the objective function (utility function):

$$
\pi(\boldsymbol{w})=\boldsymbol{\mu}^{\prime} \boldsymbol{w}-\frac{\gamma}{2} \boldsymbol{w}^{\prime} \boldsymbol{\Sigma} \boldsymbol{w}
$$

Let $\boldsymbol{w}(\boldsymbol{\mu}, \boldsymbol{\Sigma})$ be a true (but unknown) optimal portfolio weight based on parameters $\boldsymbol{\mu}$ and $\boldsymbol{\Sigma}$, if $\hat{\boldsymbol{\mu}}$ and $\hat{\boldsymbol{\Sigma}}$ denote the estimators of on $\boldsymbol{\mu}$ and $\boldsymbol{\Sigma}$, respectively, and let $\hat{\boldsymbol{w}}(\hat{\boldsymbol{\mu}}, \hat{\boldsymbol{\Sigma}})$ be an estimator for optimal portfolio weights based on the estimators $\hat{\boldsymbol{\mu}}$ and $\hat{\boldsymbol{\Sigma}}$. As stated by [10], the loss function of the estimator $\hat{\boldsymbol{w}}(\hat{\boldsymbol{\mu}}, \hat{\boldsymbol{\Sigma}})$ is given by

$$
L(\boldsymbol{w} ; \hat{\boldsymbol{w}})=\pi(\boldsymbol{w})-\pi(\hat{\boldsymbol{w}}) .
$$


Some researchers have adopted the similar loss function, for example Mori [14], Kan and Zhou [9], Okhrin and Schmid [15] and Golosnoy and Okhrin [7]. The problem of estimation error in the optimal portfolio weights can be measured by taking the expected loss function, which is defined as

$$
R(\boldsymbol{w} ; \hat{\boldsymbol{w}})=E(L(\boldsymbol{w} ; \hat{\boldsymbol{w}}))=E(\pi(\boldsymbol{w})-\pi(\hat{\boldsymbol{w}})) .
$$

According to Kinkawa [10], equation (7) is also called the risk function. An estimator $\hat{\boldsymbol{w}}_{1}\left(\hat{\boldsymbol{\mu}}_{1}, \hat{\boldsymbol{\Sigma}}_{1}\right)$ is said to dominate an estimator $\hat{\boldsymbol{w}}_{2}\left(\hat{\boldsymbol{\mu}}_{2}, \hat{\boldsymbol{\Sigma}}_{2}\right)$, if for all $(\boldsymbol{\mu}, \boldsymbol{\Sigma}), \quad R\left(\hat{\boldsymbol{w}}_{1}\left(\hat{\boldsymbol{\mu}}_{1}, \hat{\boldsymbol{\Sigma}}_{1}\right)\right) \leq R\left(\hat{\boldsymbol{w}}_{2}\left(\hat{\boldsymbol{\mu}}_{2}, \hat{\boldsymbol{\Sigma}}_{2}\right)\right)$ with strict inequality for some $(\boldsymbol{\mu}, \boldsymbol{\Sigma})$. So, the expected loss function measures the sensitivity of the optimization procedure to the error estimation: a low value corresponds to a high precision of the portfolio weights estimation.

Given the utility function (equation (5)), thus $\pi(\hat{w})$ is written as

$$
\begin{aligned}
\pi(\hat{\boldsymbol{w}})= & \frac{n c}{\gamma} \boldsymbol{\mu}^{\prime}\left(\boldsymbol{T}^{\prime}\right)^{-1} F_{1}\left(\boldsymbol{T}^{-1} \boldsymbol{A}, n \boldsymbol{T}^{-1} \boldsymbol{S}\left(\boldsymbol{T}^{\prime}\right)^{-1}\right)^{2} \boldsymbol{T}^{-1} \overline{\boldsymbol{r}} \\
& -\frac{\gamma}{2}\left\{\frac{n^{2} c^{2}}{\gamma^{2}}\left(\boldsymbol{T}^{\prime}\right)^{-1} F_{1}\left(\boldsymbol{T}^{-1} \boldsymbol{A}, n \boldsymbol{T}^{-1} \boldsymbol{S}\left(\boldsymbol{T}^{\prime}\right)^{-1}\right) \overline{\boldsymbol{x}}\right. \\
& +\frac{n c}{\gamma} \boldsymbol{b}^{\prime} F_{2}^{\prime}(\boldsymbol{A}, n \boldsymbol{S}) F_{1}(\boldsymbol{A}, n \boldsymbol{S}) \overline{\boldsymbol{r}} \\
& \left.+\frac{n c}{\gamma} F_{1}(\boldsymbol{A}, n \boldsymbol{S}) \overline{\boldsymbol{r}} F_{2}(\boldsymbol{A}, n \boldsymbol{S}) \boldsymbol{b}+\boldsymbol{b}^{\prime} F_{2}^{\prime}(\boldsymbol{A}, n \boldsymbol{S}) F_{2}(\boldsymbol{A}, n \boldsymbol{S}) \boldsymbol{b}\right\} \\
= & \frac{n c}{\gamma} \boldsymbol{\mu}^{\prime} F_{1}(\boldsymbol{A}, n \boldsymbol{S}) \overline{\boldsymbol{r}}-\frac{n^{2} c^{2}}{2 \gamma} \overline{\boldsymbol{x}}^{\prime} F_{1}^{\prime}(\boldsymbol{A}, n \boldsymbol{S}) \sum F_{1}(\boldsymbol{A}, n \boldsymbol{S}) \overline{\boldsymbol{r}} \\
& -n c F_{1}(\boldsymbol{A}, n \boldsymbol{S}) \overline{\boldsymbol{r}} F_{2}(\boldsymbol{A}, n \boldsymbol{S}) \boldsymbol{b}+\text { terms do not contain } c .
\end{aligned}
$$

Next, the expected utility function is:

$$
E(\pi(\hat{\boldsymbol{w}}))=\frac{n c}{\gamma}\left[\frac{\boldsymbol{\mu}^{\prime} F_{1}(\boldsymbol{A}, \boldsymbol{\Sigma}) \boldsymbol{\mu}}{(n-p+q-2)}\right]
$$


The Optimal Portfolio Weights Using the Proportional Type ...

$$
\begin{aligned}
& -\frac{n^{2} c^{2}}{2 \gamma}\left[\frac{(n-2) \boldsymbol{\mu}^{\prime} F_{1}(\boldsymbol{A}, \boldsymbol{\Sigma}) \boldsymbol{\mu}+\frac{(p-q)}{n}}{(n-p+q-1)(n-p+q-2)(n-p+q-4)}\right] \\
& + \text { terms do not contain } c \text {. }
\end{aligned}
$$

Let $s=(n-p+q-1) ; \varphi^{2}=\boldsymbol{\mu} F_{1}(\boldsymbol{A}, \boldsymbol{\Sigma}) \boldsymbol{\mu}$. Equation (8) can be written as

$$
E(\pi(\hat{w}))=\frac{n c}{\gamma}\left[\frac{\varphi^{2}}{(s-1)}\right]-\frac{n^{2} c^{2}}{2 \gamma}\left[\frac{(n-2) \varphi^{2}+\frac{(p-q)}{n}}{(s)(s-1)(s-3)}\right]
$$

+ terms do not contain $c$.

The constant $c_{p}$ maximizes $E(\pi(\hat{\boldsymbol{w}}(\hat{\boldsymbol{\mu}}, \hat{\boldsymbol{\Sigma}})))$ if $c_{p}=\frac{s(s-3)}{n(n-2)} \theta$, where $\theta=$ $\frac{\varphi^{2}}{\varphi^{2}+\frac{(p-q)}{n}}$. The maximizer $c_{p}$ depends on the unknown parameters $\boldsymbol{\mu}$ and $\boldsymbol{\Sigma}$. Instead of using $c_{p}$, we use the constant $c_{p}^{*}=\frac{s(s-3)}{n(n-2)}$. Next, we can obtain the risk expression of $\hat{\boldsymbol{w}}(\hat{\boldsymbol{\mu}}, \hat{\boldsymbol{\Sigma}})$ by plugging in equation (9) into equation (7):

$$
\begin{aligned}
R(\boldsymbol{w} ; \hat{\boldsymbol{w}})= & \left(1-\frac{2 n c}{s-1}\right) \frac{\varphi^{2}}{2 \gamma}+\frac{n^{2} c^{2}}{2 \gamma}\left[\frac{(n-2) \varphi^{2}+\frac{(p-q)}{n}}{(s)(s-1)(s-3)}\right] \\
& + \text { terms do not contain } c \text {. }
\end{aligned}
$$

The maximizer $c_{p}^{*}$ minimizes the risk of the estimator of optimal portfolio weights $\hat{\boldsymbol{w}}(\hat{\boldsymbol{\mu}}, \hat{\boldsymbol{\Sigma}})$. Therefore, $\hat{\boldsymbol{w}}\left(c_{p}^{*} ; \overline{\boldsymbol{x}}, \boldsymbol{S}\right)=\frac{c_{p}^{*}}{\gamma} F_{1}(\boldsymbol{A}, \boldsymbol{S}) \overline{\boldsymbol{x}}+F_{2}(\boldsymbol{A}, \boldsymbol{S}) \boldsymbol{b}$ dominates the unbiased estimators that correspond to the choice $c^{*}=$ $\frac{n-p+q-2}{n}$ and maximum likelihood estimator (classical) with $c=1$. 


\section{Comparison of the Proportional Type Estimator}

In previous sections, we have investigated the properties of the proportional type estimator of optimal portfolio weights analytically. In this section, the estimation will be evaluated by simulation and empirical data.

\subsection{Validation simulation}

The risk behaviors of three estimators are evaluated by Monte Carlo simulation. The three estimators are: (i) A classical estimator $\hat{\boldsymbol{w}}(c ; \overline{\boldsymbol{r}}, \boldsymbol{S})$ with $c=1$, (ii) an unbiased estimator $\hat{\boldsymbol{w}}\left(c^{*} ; \overline{\boldsymbol{r}}, \boldsymbol{S}\right)$ with $c^{*}=\frac{n-p+q-2}{n}$, (iii) a "dominant" estimator $\hat{\boldsymbol{w}}\left(c_{p}^{*} ; \overline{\boldsymbol{r}}, \boldsymbol{s}\right)$ with $c_{p}^{*}=\frac{s(s-3)}{n(n-2)}$.

Theoretically the results of Sections 2 and 3 require that time series of data be serially independent and multivariate to be normally distributed. The procedure of Monte Carlo simulation is similar to that of Kinkawa [10] and this procedure is explained in the following algorithm:

\section{Algorithm 1:}

1. Generate $n$ random vectors from $p$-variate normal distribution $\boldsymbol{r} \sim$ $\boldsymbol{N}_{\boldsymbol{p}}\left(\left(\varphi^{2} / p\right)^{1 / 2} \mathbf{1}, \boldsymbol{I}\right)$ for some selected values of $\varphi^{2}$.

2. Calculate the risk function for all estimators by using equation (10).

3. Repeat steps 1-2 to 10,000 times.

4. Calculate the average risk function for each estimator.

As can be seen from equation (10), the risk function of the proportional type estimator depends on parameters $\boldsymbol{\mu}$ and $\boldsymbol{\Sigma}$ only through $\varphi^{2}=$ $\boldsymbol{\mu} F_{1}(\boldsymbol{A}, \boldsymbol{\Sigma}) \boldsymbol{\mu}$. That is, the risk values are the same for any $\boldsymbol{\mu}$ and $\boldsymbol{\Sigma}$ if the value of $\varphi^{2,} s$ is constant.

In this simulation, we set $p=10$ and 20; $n=60,100$ and $240 ; \varphi^{2}=$ $0.05 ; 0.10 ; 0.5$ and 1 , while $\gamma=3$. The result of simulation study can be seen on Table 1. 
The Optimal Portfolio Weights Using the Proportional Type ... 651

Table 1. Risk values of estimators for optimal portfolio weights for some pairs of $p$ and $n$

\begin{tabular}{|c|c|c|c|c|}
\hline \multirow[b]{2}{*}{$p=10, n=60$} & \multicolumn{4}{|c|}{ Risk (\%) } \\
\hline & $\varphi^{2}=0.05$ & $\varphi^{2}=0.10$ & $\varphi^{2}=0.5$ & $\varphi^{2}=1$ \\
\hline$\hat{\boldsymbol{w}}(c ; \overline{\boldsymbol{x}}, \boldsymbol{S})$ & 33.7430 & 33.7101 & 33.7927 & 33.7702 \\
\hline$\hat{\boldsymbol{w}}\left(c^{*} ; \overline{\boldsymbol{x}}, \boldsymbol{S}\right)$ & 21.9011 & 21.1882 & 21.9295 & 21.9166 \\
\hline$\hat{\boldsymbol{w}}\left(c_{p}^{*} ; \overline{\boldsymbol{x}}, \boldsymbol{S}\right)$ & 15.5129 & 15.4972 & 15.5436 & 15.5257 \\
\hline \multicolumn{5}{|l|}{$p=10, n=100$} \\
\hline$\hat{\boldsymbol{w}}(c ; \overline{\boldsymbol{x}}, \boldsymbol{S})$ & 14.2493 & 14.2527 & 14.2488 & 14.2551 \\
\hline$\hat{\boldsymbol{w}}\left(c^{*} ; \overline{\boldsymbol{x}}, \boldsymbol{S}\right)$ & 11.1656 & 11.1682 & 11.1654 & 11.1699 \\
\hline$\hat{\boldsymbol{w}}\left(c_{p}^{*} ; \overline{\boldsymbol{x}}, \boldsymbol{S}\right)$ & 9.1034 & 9.1056 & 9.1030 & 9.1071 \\
\hline \multicolumn{5}{|l|}{$p=10, n=240$} \\
\hline$\hat{\boldsymbol{w}}(c ; \overline{\boldsymbol{x}}, \boldsymbol{S})$ & 4.4973 & 4.4981 & 4.4966 & 4.4976 \\
\hline$\hat{\boldsymbol{w}}\left(c^{*} ; \overline{\boldsymbol{x}}, \boldsymbol{S}\right)$ & 4.0863 & 4.0869 & 4.0856 & 4.0866 \\
\hline$\hat{\boldsymbol{w}}\left(c_{p}^{*} ; \overline{\boldsymbol{x}}, \boldsymbol{S}\right)$ & 3.7567 & 3.7574 & 3.7561 & 3.7570 \\
\hline \multicolumn{5}{|l|}{$p=20, n=60$} \\
\hline$\hat{\boldsymbol{w}}(c ; \overline{\boldsymbol{x}}, \boldsymbol{S})$ & 187.6827 & 188.6914 & 188.0873 & 187.8029 \\
\hline$\hat{\boldsymbol{w}}\left(c^{*} ; \overline{\boldsymbol{x}}, \boldsymbol{S}\right)$ & 73.4808 & 73.8268 & 73.6196 & 73.5220 \\
\hline$\hat{\boldsymbol{w}}\left(c_{p}^{*} ; \overline{\boldsymbol{x}}, \boldsymbol{S}\right)$ & 37.1301 & 37.3564 & 37.2209 & 37.1570 \\
\hline \multicolumn{5}{|l|}{$p=20, n=100$} \\
\hline$\hat{w}(c ; \bar{x}, S)$ & 49.7336 & 49.7722 & 49.7843 & 49.7216 \\
\hline$\hat{\boldsymbol{w}}\left(c^{*} ; \overline{\boldsymbol{x}}, \boldsymbol{S}\right)$ & 29.9895 & 30.0101 & 30.0165 & 29.9831 \\
\hline$\hat{\boldsymbol{w}}\left(c_{p}^{*} ; \overline{\boldsymbol{x}}, \boldsymbol{S}\right)$ & 19.9790 & 19.9954 & 20.0005 & 19.9739 \\
\hline \multicolumn{5}{|l|}{$p=20, n=240$} \\
\hline$\hat{w}(c ; \bar{x}, S)$ & 11.4114 & 11.4088 & 11.4156 & 11.4102 \\
\hline$\hat{\boldsymbol{w}}\left(c^{*} ; \overline{\boldsymbol{x}}, \boldsymbol{S}\right)$ & 9.4356 & 9.4337 & 9.4389 & 9.4347 \\
\hline$\hat{\boldsymbol{w}}\left(c_{p}^{*} ; \overline{\boldsymbol{x}}, \boldsymbol{S}\right)$ & 7.9768 & 7.9750 & 7.9798 & 7.9760 \\
\hline
\end{tabular}


From the results given in Table 1, we see that the risks of all estimators decrease when the number of observations increases. In this case, we find that the "dominant" estimator $\hat{\boldsymbol{w}}\left(c_{p}^{*} ; \overline{\boldsymbol{r}}, \boldsymbol{S}\right)$ has a smaller risk than the unbiased estimator $\hat{\boldsymbol{w}}(c ; \overline{\boldsymbol{r}}, \boldsymbol{S})$. Therefore, $\hat{\boldsymbol{w}}\left(c_{p}^{*} ; \overline{\boldsymbol{r}}, \boldsymbol{S}\right)$ is more effective compared to the other estimators because a low value of risk corresponds to a high precision of the portfolio weights.

\subsection{Empirical verification}

Data used in this study are collected from Jakarta Stocks Exchange (JSE) consisting of 10 companies categorized as the blue chip namely AKRA = Akr Corporindo, BBRI = Bank Rakyat Indonesia, BMRI = Bank Mandiri (Persero), INDF = Indofood SuksesMakmur, INTP = Indocement Tunggal Prakarsa, JSMR = JasaMarga, LPKR = LippoKarawaci, MNCN = Media Nusantara Citra, SMGR = Semen Indonesia, and UNVR = Unilever Indonesia. As revealed by the New York Stock Exchange, a blue chip is stocked in a corporation with a national reputation for quality, reliability, and ability to operate profitably in good and bad times. Our time series data span was from 12/09/2012 to 29/12/2014 with a total of 120 weekly returns.

In Subsection 4.1, we examine the risk behavior of proportional type estimators under the normality and independence assumptions. Here, we apply the $Q-Q$ normal plot to check the validity of a distributional assumption for the data. Figure 1 displays the $Q-Q$ normal plot for the data returns. 


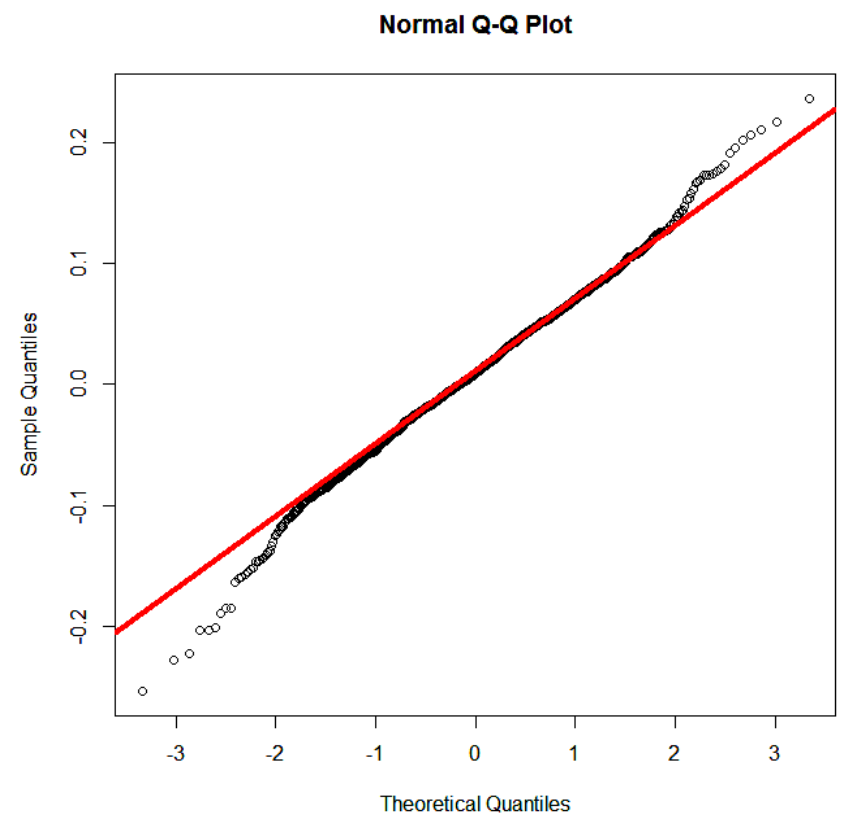

Figure 1. $Q-Q$ normal plot for data returns.

The data in this $Q-Q$ plot appear to be normally distributed, because even though there is a slight, possibly curved trend in the plot, the circles are still plenty close enough to the line to not disqualify these data from being normal.

Empirical time series of asset returns do not satisfy the assumption of independence. Therefore, we use a block bootstrap to handle this issue. A standard bootstrapping technique with sample single returns breaks the dependence between consecutive samples. We employ a moving block bootstrap method, which samples block of data preserving the dependence structure. The implementation of the block bootstrap method in our work is similar to that in Palczewski and Palczewski [16].

In this section, we compare the performance of the three estimators: $\hat{\boldsymbol{w}}(c ; \overline{\boldsymbol{r}}, \boldsymbol{S}) ; \hat{\boldsymbol{w}}\left(c^{*} ; \overline{\boldsymbol{r}}, \boldsymbol{S}\right)$ and $\hat{\boldsymbol{w}}\left(c_{p}^{*} ; \overline{\boldsymbol{r}}, \boldsymbol{S}\right)$ based on the ex post excess returns average, standard deviation, Sharpe ratio and utility. We use a rolling horizon procedure similar to that in DeMiguel and Nogales [5] and DeMiguel et al. 
[6] to compare the performance of the estimators. The detail of the method can be defined as follows:

\section{Algorithm 2:}

1. Set the length of estimation window $k$ where $k<n$.

2. Given the first window, do the moving block bootstrap.

3. Compute the optimal portfolio weight for each strategy, call it $\hat{\boldsymbol{w}}(c ; \overline{\boldsymbol{r}}, \boldsymbol{S}), \hat{\boldsymbol{w}}\left(c^{*} ; \overline{\boldsymbol{r}}, \boldsymbol{S}\right)$ and $\hat{\boldsymbol{w}}\left(c_{p}^{*} ; \overline{\boldsymbol{r}}, \boldsymbol{S}\right)$.

4. Calculate ex post excess returns in period $t+1$ i.e., $\hat{r}_{t+1}=\left(\hat{w}_{t}\right)^{\prime} r_{t+1}$.

5. Repeat steps 2-4 for the next window by including the next data point and drop the first data point of the estimation window (we assumed that investors would rebalance their portfolios for every week).

After collecting the time series of the excess returns $\hat{r}_{t+1}$, then calculate ex post average

$$
\hat{\mu}^{e x}=\frac{1}{n-k} \sum_{t=k}^{n-1} \hat{\boldsymbol{w}}_{t}^{\prime} \boldsymbol{r}_{t+1} ; \quad \hat{\sigma}^{e x}=\sqrt{\frac{1}{n-k-1} \sum_{t=k}^{n-1}\left(\hat{\boldsymbol{w}}_{t}^{\prime} r_{t+1}-\hat{\mu}^{e x}\right)^{2}} ;
$$

Sharpe ratio $S R^{e x}=\frac{\hat{\mu}^{e x}}{\hat{\sigma}^{e x}}$ and utility $\hat{\pi}^{e x}=\hat{\mu}^{e x}-\frac{\gamma}{2} \hat{\sigma}^{e x}$. To perform this empirical study, we need to set several values. First we set risk aversion $\gamma=3$ similar to that in Kinkawa [10]. Next, the length of estimation window (k) equal to 60 and 100. Table 2 presents average, standard deviation and Sharpe ratio of ex post returns for all estimators. The utility $\hat{\pi}^{e x}$ used to measure the effectiveness of estimators. 
Table 2. The performance of ex post average, standard deviation, Sharpe ratio and utility for all estimators

\begin{tabular}{|c|c|c|c|c|c|c|c|c|}
\hline & \multicolumn{4}{|c|}{$k=60$} & \multicolumn{4}{|c|}{$k=100$} \\
\hline & $\begin{array}{l}\hat{\mu}^{e x} \\
(\%)\end{array}$ & $\begin{array}{l}\hat{\sigma}^{e x} \\
(\%)\end{array}$ & $S R^{e x}$ & $\hat{\pi}^{e x}$ & $\begin{array}{l}\hat{\mu}^{e x} \\
(\%)\end{array}$ & $\begin{array}{l}\hat{\sigma}^{e x} \\
(\%)\end{array}$ & $S R^{e x}$ & $\hat{\pi}^{e x}$ \\
\hline$\hat{\boldsymbol{w}}(c ; \overline{\boldsymbol{x}}, \boldsymbol{S})$ & 0.431 & 10.249 & 0.042 & -0.149 & 1.662 & 6.627 & 0.251 & -0.083 \\
\hline$\hat{\boldsymbol{w}}\left(c^{*} ; \overline{\boldsymbol{x}}, \boldsymbol{S}\right)$ & 0.484 & 9.454 & 0.051 & -0.137 & 1.599 & 6.149 & 0.260 & -0.076 \\
\hline$\hat{\boldsymbol{w}}\left(c_{p}^{*} ; \overline{\boldsymbol{x}}, \boldsymbol{S}\right)$ & 0.529 & 8.796 & 0.060 & -0.127 & 1.546 & 5.756 & 0.269 & -0.071 \\
\hline
\end{tabular}

We see that in all cases, the "dominant" estimator $\hat{\boldsymbol{w}}\left(c_{p}^{*} ; \overline{\boldsymbol{r}}, \boldsymbol{S}\right)$ has smaller ex post standard deviation. Also, we can investigate that estimator $\hat{\boldsymbol{w}}\left(c_{p}^{*} ; \overline{\boldsymbol{r}}, \boldsymbol{S}\right)$ is outperformed compared to other estimators, since it generates the larger ex post Sharpe ratio and utility. Despite the performance of ex post average, the estimator $\hat{\boldsymbol{w}}\left(c_{p}^{*} ; \overline{\boldsymbol{r}}, \boldsymbol{S}\right)$ has the largest ex post average only in the case $k=60$. Thus, we find that the proportional type estimator using $c=c_{p}^{*}$ is effective for these data set.

\section{Conclusion}

We have evaluated the proportional type estimators for the meanvariance optimal portfolio weights analytically when the mean vector and covariance matrix are unknown. The sensitivity of portfolio weights is measured by the expected loss function (the risk function) which combines error estimation of portfolio weights into a single number. This facilitates the comparison of various estimations on the proportional type estimator.

The theoretical results are complemented by a thorough simulation with varying sizes data sets and ex post excess returns of empirical study. We show that the risk of estimators computed by simulation study is in good agreement with the theory. In the class of proportional type estimator, we find that the "dominant" estimator $\hat{\boldsymbol{w}}\left(c_{p}^{*} ; \overline{\boldsymbol{r}}, \boldsymbol{S}\right)$ is more effective than the unbiased estimator $\hat{\boldsymbol{w}}(c ; \overline{\boldsymbol{r}}, \boldsymbol{S})$. Furthermore, the empirical results also show 
that the estimator $\hat{\boldsymbol{w}}\left(c_{p}^{*} ; \overline{\boldsymbol{r}}, \boldsymbol{S}\right)$ significantly outperformed in terms of average, standard deviation, Sharpe ratio and utility of ex post excess returns.

We believe that the expected loss function (the risk function) should gain wider popularity as a tool for assessing properties of portfolio estimators. Further research could attempt to provide an analysis of portfolio weights error estimation for other models.

\section{References}

[1] M. J. Best and R. R. Grauer, On the sensitivity of mean-variance efficient portfolios to changes in asset means: some analytical and computational results, Rev. Fin. Stud. 4(2) (1991), 315-342.

[2] M. Broadie, Computing efficient frontiers using estimated parameters, Ann. Oper. Res. 45 (1993), 21-58.

[3] S. Ceria and R. A. Stubbs, Incorporating estimation errors into port folio selection: robust portfolio construction, J. Asset Manage. 7 (2006), 109-127.

[4] V. K. Chopra and W. T. Ziemba, The effects of errors in means, variances, and covariances on optimal portfolio choice, J. Portfolio Manage. 19(2) (1993), 6-11.

[5] V. DeMiguel and F. J. Nogales, Portfolio selection with robust estimation, J. Oper. Res. 57(3) (2009), 560-577.

[6] V. DeMiguel, A. Martin-Utrera and F. J. Nogales, Size matters: optimal calibration of shrinkage estimators for portfolio selection, J. Bank. Fin. 37 (2013), 3018-3034.

[7] V. Golosnoy and Y. Okhrin, Multivariate shrinkage for optimal portfolio weights, European J. Fin. 13(5) (2007), 441-458.

[8] J. D. Jobson and B. Korkie, Estimation of Markowitz efficient portfolios, J. Amer. Stat. Assoc. 75 (1980), 544-554.

[9] R. Kan and G. Zhou, Optimal portfolio choice with parameter uncertainty, J. Fin. Quant. Anal. 42 (2007), 717-727.

[10] T. Kinkawa, Estimation of optimal portfolio weights using shrinkage technique, Dissertation, School of Science and Technology, Keio University, 2010.

[11] O. Ledoit, Essays on risk and return in the stock Market, Doctoral Theses, Massachusetts Institute of Technology, 1995. 
[12] H. Markowitz, Portfolio selection, J. Fin. 7(1) (1952), 77-91.

[13] R. O. Michaud, The Markowitz optimization enigma: is optimized optimal?, Fin. Anal. J. 45(1) (1989), 31-42.

[14] H. Mori, Finite sample properties of estimators for the optimal portfolio weight, J. Japan Stat. Soc. 34(1) (2004), 27-46.

[15] Y. Okhrin and W. Schmid, Distributional properties of portfolio weights, J. Econ. 134(1) (2006), 235-256.

[16] A. Palczewski and J. Palczewski, Theoretical and empirical estimates of meanvariance portfolio sensitivity, European J. Oper. Res. 234(2) (2014), 402-410. 